\title{
Incidence rate and characteristics of symptomatic vitamin D deficiency in children: a nationwide survey in Japan
}

\author{
Takuo Kubota ${ }^{1), 21)}$, Hirofumi Nakayama1),21), Taichi Kitaoka1),21), Yosikazu Nakamura' ${ }^{2), 21)}$, Seiji Fukumoto ${ }^{3), 21),}$ \\ Ikuma Fujiwara ${ }^{4), 21)}$, Yukihiro Hasegawa(5),21), Kenji Ihara'),21), Sachiko Kitanaka ${ }^{7), 21)}$, Satomi Koyama ${ }^{8), 21)}$, \\ Satoshi Kusuda ${ }^{9), 21)}$, Haruo Mizuno ${ }^{10), 11), 21)}$, Keisuke Nagasaki ${ }^{12), 21)}$, Koji Oba ${ }^{13), 21)}$, Yuko Sakamoto ${ }^{14), 21)}$, \\ Noriyuki Takubo ${ }^{15), 21)}$, Toshiaki Shimizu ${ }^{15), 21)}$, Yusuke Tanahashi ${ }^{16), 21)}$, Kosei Hasegawa ${ }^{17), 21)}$, Hirokazu Tsukahara ${ }^{18), 21)}$, \\ Tohru Yorifuji ${ }^{19), 21)}$, Toshimi Michigami ${ }^{20), 21)}$ and Keiichi Ozono ${ }^{1), 21)}$
}

1) Department of Pediatrics, Osaka University Graduate School of Medicine, Osaka 565-0871, Japan

2) Department of Public Health, Jichi Medical University, Tochigi 329-0498, Japan

3) Fujii Memorial Institute of Medical Sciences, Institute of Advanced Medical Sciences, Tokushima University, Tokushima 770-8503, Japan

4) Department of Pediatric Endocrinology and Environmental Medicine, Tohoku University Graduate School of Medicine, Miyagi 980-8575, Japan

5) Division of Endocrinology and Metabolism, Tokyo Metropolitan Children's Medical Center, Tokyo 183-8561, Japan

6) Department of Pediatrics, Oita University Faculty of Medicine, Oita 879-5593, Japan

7) Department of Pediatrics, The University of Tokyo Graduate School of Medicine, Tokyo 113-8655, Japan

8) Department of Pediatrics, Dokkyo Medical University, Tochigi 321-0293, Japan

9) Department of Pediatrics, Kyorin University, Tokyo 181-0004, Japan

10) Department of Pediatrics and Neonatology, Nagoya City University Graduate School of Medical Sciences, Aichi 467-8602, Japan

11) Department of Pediatrics, International University of Health and Welfare, School of Medicine, Chiba 286-8686, Japan

12) Division of Pediatrics, Department of Homeostatic Regulation and Development, Niigata University Graduate School of Medical and Dental Sciences, Niigata 951-8520, Japan

13) Department of Biostatistics, School of Public Health, Graduate School of Medicine, The University of Tokyo, Tokyo 113-0033, Japan

14) Department of Orthopedics, Juntendo University Nerima Hospital, Tokyo 177-8521, Japan

15) Department of Pediatrics and Adolescent Medicine, Juntendo University Graduate School of Medicine, Tokyo 113-8431, Japan

16) Department of Pediatrics, Asahikawa Medical University, Hokkaido 078-8510, Japan

17) Department of Pediatrics, Okayama University Hospital, Okayama 700-8558, Japan

18) Department of Pediatrics, Okayama University Graduate School of Medicine, Dentistry and Pharmaceutical Sciences, Okayama 700-8558, Japan

19) Division of Pediatric Endocrinology and Metabolism, Children's Medical Center, Osaka City General Hospital, Osaka 534-0021, Japan

20) Department of Bone and Mineral Research, Research Institute, Osaka Women's and Children's Hospital, Osaka 594-1101, Japan

21) Study group of Survey and Estimation of Incidence Rate of Childhood Symptomatic Vitamin D

\begin{abstract}
There is concern that vitamin D deficiency is prevalent among children in Japan as well as worldwide. We conducted a nationwide epidemiologic survey of symptomatic vitamin D deficiency to observe its incidence rate among Japanese children. A questionnaire inquiring the number of new patients with vitamin D deficiency rickets and/or hypocalcemia for 3 years was sent to 855 randomly selected hospitals with a pediatrics department in Japan. In this survey, we found that 250 children were diagnosed with symptomatic vitamin D deficiency. The estimated number of patients with symptomatic vitamin D deficiency per year was $183(95 \%$ confidence interval (CI): 145-222). The overall annual incidence rate among children under 15 years of age was 1.1 per 100,000 population (95\% CI: 0.9-1.4). The second survey has provided detailed information on 89 patients with symptomatic vitamin D deficiency under 5 years of age in hospitals in the current research group. The nationwide and second surveys estimated the overall annual incidence rate of symptomatic vitamin D deficiency in children under 5 years of age to be $3.5(2.7-4.2)$ per 100,000 population. The second survey revealed $83 \%$ had bowed legs, $88 \%$ had exclusive breastfeeding, $49 \%$ had a restricted and/or unbalanced diet and $31 \%$ had insufficient sun exposure among the 89 patients. This is the first nationwide survey on definitive clinical vitamin D deficiency in children in Japan. Elucidating the frequency and characteristics of symptomatic vitamin D deficiency among children is useful to develop preventative public health strategies.
\end{abstract}

Key words: Vitamin D deficiency, Rickets, Hypocalcemia, Children, Nationwide survey

Submitted Jan. 10, 2018; Accepted Feb. 14, 2018 as EJ18-0008

Released online in J-STAGE as advance publication Mar. 10, 2018

Correspondence to: Keiichi Ozono, MD, PhD, Department of Pediatrics, Osaka University Graduate School of Medicine, 2-2 Yamadaoka, Suita, Osaka 565-0871, Japan.

E-mail: keioz@ped.med.osaka-u.ac.jp
VITAMIN D is essential for skeletal development in children, and is synthesized in the skin after exposure to ultraviolet B rays and obtained from limited foods [1]. Vitamin D deficiency causes rickets and hypocalcemic seizures. Vitamin D deficiency rickets is characterized by defective mineralization of bones and cartilage of the 
Table 1 Diagnosis of exclusion of vitamin D deficiency

hypophosphatemic rickets

hypophosphatasia

vitamin D dependency

metaphyseal dysplasias

Blount disease

hypoparathyroidism

pseudohypoparathyroidism

cholestasis

transient hyperphosphatasemia

metabolic bone disease of prematurity

growth plate [2]. Vitamin D deficiency is based on low circulating 25-hydroxyvitamin D $(25(\mathrm{OH}) \mathrm{D})$ concentration, a metabolite of vitamin $\mathrm{D}$ and the best clinical indicator of vitamin D repletion in the body. Symptomatic vitamin D deficiency presents with symptoms and signs related to rickets and/or hypocalcemia in addition to low serum 25(OH)D levels [3]. However, children with hypovitaminosis D do not always exhibit symptoms or signs associated with vitamin D deficiency $[4,5]$.

Reported patients with symptomatic vitamin D deficiency have been increasing among children in recent years throughout the world [6-9], including Japan [10-13]. Vitamin D deficiency remains a significant public health problem in the world [2] and in Japan. However, no nationwide survey of the incidence rate of symptomatic vitamin D deficiency among Japanese children has been reported, although a survey on vitamin D deficiency rickets in Hokkaido, a northern area of Japan, has been carried out [11]. Therefore, we conducted a nationwide epidemiologic survey of symptomatic vitamin D deficiency to clarify its incidence rate among Japanese children.

\section{Materials and Methods}

\section{Subjects}

Subjects were patients with symptomatic vitamin D deficiency rickets and/or hypocalcemia meeting the guidelines provided by the Japanese Society for Pediatric Endocrinology (http://jspe.umin.jp/medical/files/_vitaminD. pdf) (In Japanese). Diagnostic criteria for vitamin D deficiency rickets include the following items: Clinical symptoms and/or signs related to rickets, including deformities of lower extremities, gait abnormality, spinal curvature, craniotabes, enlarged anterior fontanel, rachitic rosary, Harrison's groove, swelling of joints, pathological fracture and growth retardation; radiographic findings related to rickets, including irregularity, cupping, splaying, fraying of the metaphysis; increased serum alkaline phosphatase (ALP) levels; increased plasma parathyroid hormone (PTH) concentrations; and either hypophosphatemia or hypocalcemia. Diagnostic criteria for vitamin D deficiency hypocalcemia include the following items: Clinical symptoms and/or signs related to hypocalcemia, including seizure, tetany, irritability, Trousseau sign and Chvostek sign; clinical symptoms and/or signs related to rickets as shown above; radiographic findings related to rickets as shown above; hypocalcemia; increased serum ALP levels; and increased plasma PTH concentrations. Decreased serum 25(OH)D concentrations were not included in the criteria in this study because the measurement of $25(\mathrm{OH}) \mathrm{D}$ was not approved by the national health insurance system in Japan at the time when the subjects visited hospitals. However, serum 25(OH)D levels were measured in most patients as a result of this survey. Exclusion criteria are summarized in Table 1.

\section{Methods}

According to the Nationwide Epidemiologic Survey Manual issued by The Epidemiological Study Group of Specified Rare and Intractable Diseases, a questionnaire inquiring the number of new patients with vitamin D deficiency rickets and/or hypocalcemia visiting the target hospitals between April 1, 2013 and March 31, 2016 was sent to 855 hospitals (32\%) with a pediatrics department, which were randomly selected from all hospitals $(2,677)$ in Japan (Table 2). The selection rate was determined based on stratification by the number of beds in the hospitals; the more beds a hospital had, the higher the probability of being selected. The selection rate was $100 \%$ for hospitals with 500 beds or more and medical university hospitals, whereas it was 5\% for hospitals with 99 beds or less at random. We previously estimated the incidence of X-linked hypophosphatemic rickets in Japan in a similar survey [14].

Considering the selection rate and response rate to the survey, the total number of patients with symptomatic vitamin D deficiency was estimated as follows. The formula for the estimation of the patient number in each stratum: The estimated number of patients $=$ the reported number of patients/(selection rate $\times$ response rate); the numbers of patients in each stratum were summed. The 
Table 2 Three-year estimated number of patients with symptomatic vitamin D deficiency

\begin{tabular}{|c|c|c|c|c|c|c|c|c|c|c|}
\hline \multirow{3}{*}{$\begin{array}{l}\text { Classification (beds) } \\
\text { Univ. Hosp. }\end{array}$} & \multicolumn{3}{|c|}{ Hospital No. } & \multicolumn{4}{|c|}{ Visited Patient No. } & \multicolumn{3}{|c|}{ Estimated Patient No. } \\
\hline & All & Targeted & Responded & Rickets & Hypocalcemia & Both & Total & Estimate & & \\
\hline & 133 & 133 & 78 & 106 & 12 & 8 & 126 & 215 & 165 & 269 \\
\hline $500-$ & 232 & 232 & 121 & 55 & 7 & 1 & 63 & 121 & 92 & 149 \\
\hline $400-499$ & 223 & 178 & 102 & 29 & 3 & 2 & 34 & 74 & 11 & 140 \\
\hline $300-399$ & 349 & 140 & 84 & 21 & 4 & 0 & 25 & 104 & 49 & 159 \\
\hline $200-299$ & 341 & 68 & 36 & 1 & 0 & 0 & 1 & 9 & -8 & 27 \\
\hline $100-199$ & 665 & 67 & 25 & 1 & 0 & 0 & 1 & 27 & -24 & 77 \\
\hline-99 & 734 & 37 & 12 & 0 & 0 & 0 & 0 & 0 & 0 & 0 \\
\hline Total & 2,677 & 855 & 458 & 213 & 26 & 11 & 250 & 550 & 434 & 666 \\
\hline
\end{tabular}

95\% confidence interval (CI) was calculated based on multinomial hypergeometric distribution [15].

A second survey investigated detailed data for patients with symptomatic vitamin D deficiency in the pediatrics departments of 9 hospitals in the current research group. Subjects were new patients who visited the hospitals in a 3.5-year period from April 1, 2013 to September 30, 2016. The 9 hospitals consisted of university or children's hospitals with over 500 beds. The diagnosis of symptomatic vitamin D deficiency was based on pediatricians' determination at the local hospitals in the second survey. Clinical presentation, age, feeding before solid food, restricted and/or unbalanced diet, sunlight exposure, race, biochemical data and treatment were evaluated. This study was approved by the institutional ethical committee of Osaka University Hospital (approval number 16040).

\section{Results}

\section{Incidence of symptomatic vitamin D deficiency}

The response rate to the survey was $53.0 \%(458 / 855$ hospitals). The hospitals reported 250 patients newly diagnosed with symptomatic vitamin D deficiency during the 3 years, comprising 213 patients with vitamin D deficiency rickets, 26 with vitamin $\mathrm{D}$ deficiency hypocalcemia and 11 with both (Table 2). Serum 25(OH)D levels were measured in $90 \%(226 / 250)$ of patients and were less than $20 \mathrm{ng} / \mathrm{mL}$ in all. The estimated number of patients with vitamin $\mathrm{D}$ deficiency for the 3-year period was 550 (95\% CI: 434-666) by summing the estimated number of patients in each stratum based on the hospital size. Thus, the estimated annual number of patients with vitamin D deficiency was 183 (95\% CI: 145-222). The population under the age of 15 years was $16,233,000$ in 2014. Thus, the overall annual incidence rate of symptomatic vitamin D deficiency in children under 15 years of age in Japan was 1.1 (95\% CI: 0.9-1.4) per 100,000 children.

The second survey revealed 106 patients with symptomatic vitamin D deficiency. The following 17 patients were excluded due to underlying diseases, limited manifestations or insufficient data: 3 patients with mental retardation; 2, cholestasis; 2, 21 trisomy; 1 , citrin deficiency; 1, neuroblastoma; 1, TRPV6 compound heterozygous mutations; 1 , preterm birth, low birth weight and regular follow-ups; 5, neither radiographic changes of rickets nor symptomatic hypocalcemia; 1, insufficient data. The remaining 89 patients were analyzed: 80 with vitamin D deficiency rickets, 6 with vitamin D deficiency hypocalcemia and 3 with both. The median age was 1.75 years [1.50-2.33] (median [interquartile range (IQR)]). Patients under 5 years of age accounted for $98.9 \%(88 / 89)$ in the second survey. We then estimated the annual incident rate of symptomatic vitamin D deficiency in younger children. When the age ratio (98.9\%) in the second survey was applied to the first nationwide survey (estimated annual number of patients was 183 [95\% CI: 145-222]), the estimated annual number of patients under 5 years of age was 181 (143-220). The population under the age of 5 years was 5,214,000 in 2014. Thus, the overall annual incidence rate of symptomatic vitamin $\mathrm{D}$ deficiency in children under 5 years of age is $3.5(2.7-4.2)$ per 100,000 population. Similarly, the overall annual incidence rate of symptomatic vitamin D deficiency in children under 3 years of age is 5.4 (4.36.5) per 100,000 population because the age ratio of those under 3 years of age in the second survey was 
$92.1 \%$ and the population under the age of 3 years was $3,103,000$.

\section{Characteristics of symptomatic vitamin D deficiency}

Clinical features, including presentation, feeding before solid foods, restricted and/or unbalanced diet, sunlight exposure, race, biochemical data and treatment were evaluated in the second survey. Chief presentations consisted of bowed legs ( $n=74,83 \%)$, short stature ( $n=$ $18,20 \%)$, gait abnormalities ( $n=15,17 \%)$, screening $(n$ $=10,11 \%)$, convulsions $(n=4,4 \%)$ and others $(n=6$, $7 \%$ ) (Fig. 1). Exclusive breastfeeding ( $n=78,88 \%$ ) was dominant before solid food compared with breast plus formula feeding $(n=7,8 \%)$ and exclusive formula feeding $(n=1,1 \%)$. Information on vitamin $\mathrm{D}$ supplementation was not available. Contributing factors to vitamin D deficiency were restricted and/or unbalanced diet in 44 patients and insufficient sun exposure in 28. Dietary problems consisted of food allergy in 26 patients, meal preference in 10, prolonged breast-feeding in 4 and vegetarianism in 3. Diet restrictions due to food allergy or self-determination included eggs for 40 patients, dairy products for 32, wheat for 9 and soybeans for 6 . Insufficient sun exposure was due to infrequent outdoor playing for 19 patients and wearing sun screen for 9. Most patients were Japanese $(n=86,97 \%)$; one parent of the others $(n=3)$ was African, American or half-Japanese and half-Costa Rican, respectively. X-ray changes, including long bone deformities and rachitic findings, were present in 85 (96\%) among 89 patients. Biochemical data for children with symptomatic vitamin D deficiency are summarized in Table 3. Serum 25(OH)D levels were $9(6.0-13.8) \mathrm{ng} / \mathrm{mL}$ (median [IQR]) and below $20 \mathrm{ng} / \mathrm{mL}$ in $96 \%$ of patients. During treatment, 83 patients received alfacalcidol at the maximum dose of $0.10(0.07-0.12) \mu \mathrm{g} / \mathrm{kg} /$ day (median [IQR]) and 18 patients received calcium lactate at the maximum dose of $100(71-175) \mathrm{mg} / \mathrm{kg} / \mathrm{day}$.

\section{Discussion}

To the best of our knowledge, this is the first nationwide survey on definitive clinical vitamin D deficiency in children in Japan. This study was based on a hospital survey and included patients with symptomatic vitamin D deficiency, not only low serum 25(OH)D levels. Approximately 183 children without underlying illness develop symptomatic vitamin D deficiency per year in Japan, resulting in the overall annual incidence rate of

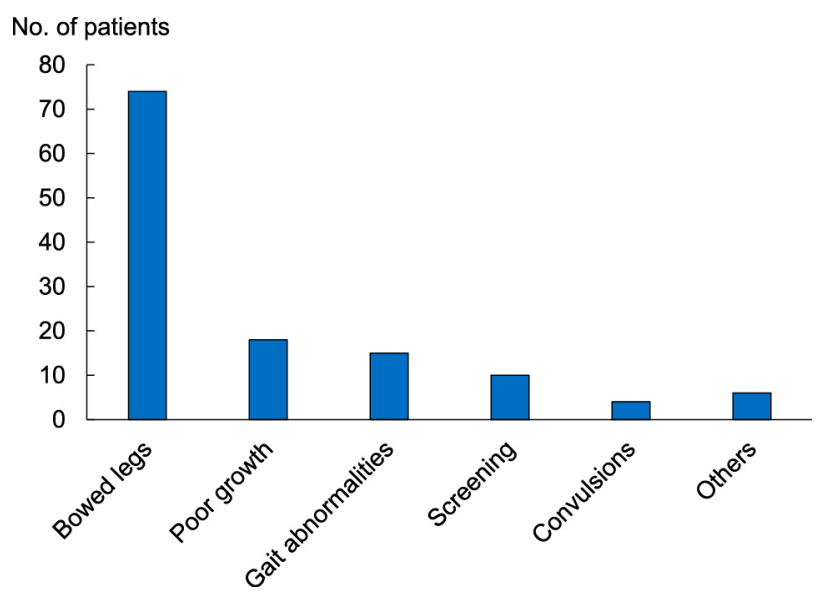

Fig. 1 Mode of presentation of symptomatic vitamin D deficiency

1.1 per 100,000 children under 15 years of age.

We defined symptomatic vitamin D deficiency based on the guidelines provided by the Japanese Society for Pediatric Endocrinology, which include clinical, radiographic and biochemical findings associated with vitamin D deficiency. The diagnostic criteria in the current study excluded patients with "mild" or improving clinical vitamin $\mathrm{D}$ deficiency in the absence of one of the above findings, resulting in a reduction in the incidence of symptomatic vitamin D deficiency. However, definitive diagnostic criteria are crucial for the survey of the incidence of a disorder, and the criteria in the current study are comparable with other studies (Table 4).

There were few reports regarding the incidence of symptomatic vitamin D deficiency in limited regions of Japan. Matsuo $\mathrm{K}$ et al. discussed the prevalence of vitamin D deficiency rickets in Hokkaido, a northern area of Japan [11], which has the highest incidence rate of vitamin D deficiency in Japan (Table 4). An estimate of the annual incidence rate of patients with vitamin $\mathrm{D}$ deficiency rickets was 9 in 100,000 children under 4 years of age. The age of the subjects in the Hokkaido study was much younger than that in the current study. As infants and toddlers are more prone to symptomatic vitamin D deficiency than older children [16], age under 4 years led to a higher incidence rate than age under 15 years. Yorifuji $\mathrm{J}$ et al. reported the incidence of craniotabes in normal newborns in a single hospital [12], but not the exact incidence rate of vitamin D deficiency. The study found that craniotabes was present in $246(22.0 \%)$ neonates among 1,120 normal Japanese in one year, and at 1 month of age, infants with craniotabes had signifi- 
Table 3 Biochemical analysis of children with symptomatic vitamin D deficiency

\begin{tabular}{lccccc}
\hline & No. of children & Reference range & Median & Interquartile range & No. abnormal (\%) \\
\hline 25-hydroxyvitamin D (ng/mL) & 75 & $>20$ & 9.0 & $6.0-13.8$ & $72(96 \%)$ \\
Alkaline phosphatase (IU/L) & 77 & $353-1,099^{*}$ & 2,163 & $1,389-3,384$ & $68(88 \%)$ \\
Calcium (mg/dL) & 77 & $8.4-10.0$ & 9.2 & $8.3-9.6$ & $20(26 \%)$ \\
Phosphate (mg/dL) & 77 & $4.2-6.2^{*}$ & 4.0 & $3.1-5.0$ & $39(51 \%)$ \\
Parathyroid hormone (pg/mL) & 72 & $10-60$ & 199 & $105-388$ & $63(88 \%)$ \\
1,25 -dihydroxyvitamin D $(\mathrm{pg} / \mathrm{mL})$ & 53 & $20-60$ & 104 & $63-140$ & $41(77 \%)$ \\
\hline
\end{tabular}

*Reference range for the age of 1 year.

Table 4 Estimated annual incidence rate of vitamin D deficiency in children among different studies

\begin{tabular}{|c|c|c|c|c|c|c|}
\hline Country & EAIR per 100,000 & $\begin{array}{c}\text { Age } \\
\text { (years) }\end{array}$ & Clinical or radiographic findings & $\begin{array}{c}25(\mathrm{OH}) \mathrm{D} \text { levels } \\
(\mathrm{ng} / \mathrm{mL})\end{array}$ & ALP levels & Reference \\
\hline New Zealand & 2.2 & $<15$ & I. & $<20$ & High & {$[8]$} \\
\hline Canada & 2.9 & $<18$ & N.I. & $<11$ & N.I. & {$[18]$} \\
\hline Denmark $^{\#}$ & 2.9 & $<15$ & I. & $<10$ & High & [19] \\
\hline Australia & 4.9 & $<15$ & N.I. & $<20$ & High & {$[20]$} \\
\hline $\mathrm{UK}^{\#}$ & 7.5 & $<5$ & I. & N.A. & N.A. & [17] \\
\hline USA $^{\#}$ & 24 & $<3$ & I. & N.A. & N.I. & [9] \\
\hline Japan $^{\#}$ & 9 & $<4$ & I.* & $<15$ & N.I. & [11] \\
\hline This study & 1.1 & $<15$ & I. & $<20$ & High & \\
\hline
\end{tabular}

EAIR, estimated annual incidence rate; 25(OH)D, 25-hydroxyvitamin D; ALP, alkaline phosphatase; I., included; N.I., not included; N.A., not available; *, a region of the country; *, rickets alone included.

cantly higher serum ALP levels compared with normal neonates, with $37.3 \%$ having a $25(\mathrm{OH}) \mathrm{D}$ of less than 10 $\mathrm{ng} / \mathrm{mL}$. Craniotabes is one sign of symptomatic vitamin D deficiency, but plasma PTH levels and radiographic findings were not available in this study.

Several nationwide surveys on the incidence of vitamin D deficiency have been reported from some countries. The annual incidence rate of vitamin D deficiency in children varies from 2.2 to 24.1 per 100,000 children among studies $[8,9,17-20]$, which may be due to different inclusion criteria and regions (Table 4). The eligibility age for the studies in Canada, Australia, New Zealand (NZ) and Denmark was up to 18 years old, which is comparable with that in the current study. In Canada, The overall annual incidence rate was 2.9 cases per 100,000 children under the age of 18 years [18]. Most patients (89\%) were intermediate or darker skinned and some (10\%) were fair skinned. Radiographic signs of rickets were supportive of the diagnosis, but not required for inclusion. In Australia, the overall incidence in children under 15 years of age was 4.9/100,000/year [20]. Skin color was dark in $85 \%$, intermediate in $13 \%$ and fair in $2 \%$. However, not all children with vitamin D deficiency were confirmed to have radiographic rickets. In NZ, the overall annual incidence of vitamin D deficiency rickets in children aged $<15$ years was 2.2/ 100,000 (95\%CI 1.4-3.5) [8]. Most children (95\%) were born in NZ, but the majority of the mothers (68\%) were born outside NZ. In southern Denmark, the average incidence of nutritional rickets in children aged under 15 years was 2.9 per 100,000 per year [19]. Most (74\%) rickets cases occurred among immigrant children and some (26\%) among children of ethnic Danish origin. These 4 studies demonstrated higher incidence rates of vitamin D deficiency compared with that in the current study, which may be due to the lack of required radiographic findings of rickets in the Canadian and Australian studies, and the high ratio of darker or intermediate skinned and/or immigrant children in the 4 studies.

A survey study in the United Kingdom (UK) and 
United States of America (USA) had younger children with symptomatic vitamin $\mathrm{D}$ deficiency. In West Midlands County of the UK, the overall incidence of symptomatic vitamin D deficiency in children less than 5 years of age was 7.5 per 100,000 children per year [17]. Most (71\%) of the 24 patients were of South Asian ethnic origin, some (17\%) were black African or AfricanCaribbean and 1 was white. Serum levels of 25(OH)D were not considered biochemical vitamin D deficiency. In Olmsted County, Minnesota, USA, the incidence of nutritional rickets in children under 3 years was 24.1 per 100,000 [9]. Most (59\%) of the 17 patients were black, and $24 \%$ were white. The measurement of serum $25(\mathrm{OH}) \mathrm{D}$ levels was not required. The age of subjects in the UK and USA studies was much younger than that in the current study, which significantly affected the incidence rate of clinical vitamin $\mathrm{D}$ deficiency.

We performed the second survey to examine detailed data on clinical vitamin D deficiency. The patient number for analysis was 89 , equivalent to $36 \%$ of that in the nationwide survey. The classification of clinical presentation of vitamin D deficiency (i.e. rickets, hypocalcemia or both) was similar with the nationwide survey, although the diagnosis of symptomatic vitamin D deficiency was based on pediatricians' determination at the local hospitals in the second survey. The median age of 1.75 years was similar with the median and mean ages (1.4 years) in the NZ and Canadian studies [18, 21]. Collectively, the application of the age ratio in the second survey to the first nationwide survey was thought to be reasonable. Indeed, the estimated overall annual incidence rate of symptomatic vitamin D deficiency in children under 5 years of age (3.5 per 100,000 children) is $47 \%$ of that of the study in UK [17], where South Asian and black African or African-Caribbean ethnic origins are dominant in cases of symptomatic vitamin D deficiency.

The clinical features in the second survey revealed details of presentation, feeding before solid foods, diet, sunlight exposure, race, biochemical data and treatment for patients with symptomatic vitamin D deficiency and without underlying disorders in Japan. Bowed legs were the most common presentation, similar with in the UK, NZ and Canadian reports $[8,17,18]$. Exclusive breastfeeding was reconfirmed to be risk factor $[2,21]$. Notably, approximately half of the patients with symptomatic vitamin D deficiency had a restricted and/or unbalanced diet, which is consistent with the recent practical guide that restriction of cow's milk or multiple food items may increase the risk of metabolic bone disorders [22]. Unlike overseas [2, 23], active vitamin D was used to treat patients with symptomatic vitamin D deficiency in the current study. Prescribing native vitamin D, a true vitamin D replacement, should be allowed to be employed in Japan.

The limitation of the current study is the lack of data on age and ethnicity, and that not all the hospitals were covered in the nationwide survey. However, the second survey in limited hospitals with $36 \%$ of the patient number of the nationwide survey provided age data and incidence rate in children under 3 and 5 years of age. As for ethnicity, the publication by the Ministry of Justice of Japan concerning the number of foreign residents in 2014 has reported 102,087 childhood residents under 15 years of age in Japan, excluding Chinese and Koreans who are similar in skin color with Japanese (http:// www.e-stat.go.jp/SG1/estat/List.do?lid=000001133760) (In Japanese). As foreign residents aged under 15 years make up a small portion, $0.6 \%(102,087 / 16,233,000)$ in Japan, and the second survey had few darker or intermediate skinned children, ethnicity was thought to minimally influence the incidence rate of symptomatic vitamin D deficiency in the current study. Regarding the hospital selection, we randomly determined hospitals to send the questionnaire based on stratification by the number of beds in the hospitals. As expected, university hospitals or those with more beds had more patients with symptomatic vitamin D deficiency (Table 2), which indicates that the selection method in the current study was appropriate.

In conclusion, this is the first nationwide survey on symptomatic vitamin D deficiency in children in Japan. The overall incidence rate of symptomatic vitamin $\mathrm{D}$ deficiency in children less than 15 years of age was 1.1 per 100,000 children per year, and the incidence rates in those less than 3 and 5 years of age are 5.4 and 3.4, respectively. Exclusive breastfeeding and restricted and/or unbalanced diet are major risk factors for vitamin $\mathrm{D}$ deficiency. Determining the incidence rate of symptomatic vitamin D deficiency among children is useful to develop public health strategies. Symptomatic vitamin D deficiency without underlying disease is a fully preventable disorder. Thus, supplementation with vitamin D in infants and pregnant women, and food fortification with vitamin $\mathrm{D}$ and calcium should be considered to prevent symptomatic vitamin D deficiency in Japan as well as in other countries [2]. 


\section{Acknowledgements}

This work was supported in part by Grants-in-Aid from the Ministry of Health, Labour, and Welfare of Japan (H28-sukoyaka-ippan-006).

\section{Disclosures}

None of the authors have any potential conflicts of interest associated with this research.

\section{References}

1. Holick MF (2006) Resurrection of vitamin D deficiency and rickets. $J$ Clin Invest 116: 2062-2072.

2. Munns CF, Shaw N, Kiely M, Specker BL, Thacher TD, et al. (2016) Global consensus recommendations on prevention and management of nutritional rickets. $J$ Clin Endocrinol Metab 101: 394-415.

3. Elder CJ, Bishop NJ (2014) Rickets. Lancet 383: 16651676.

4. Atapattu N, Shaw N, Högler W (2013) Relationship between serum 25-hydroxyvitamin $\mathrm{D}$ and parathyroid hormone in the search for a biochemical definition of vitamin D deficiency in children. Pediatr Res 74: 552-556.

5. Tsugawa N, Uenishi K, Ishida H, Ozaki R, Takase T, et al. (2015) Association between vitamin D status and serum parathyroid hormone concentration and calcaneal stiffness in Japanese adolescents: sex differences in susceptibility to vitamin D deficiency. J Bone Miner Metab 34: 464474.

6. Cesur Y, Dogan M, Ariyuca S, Basaranoglu M, Bektas MS, et al. (2011) Evaluation of children with nutritional rickets. J Pediatr Endocrinol Metab 24: 35-43.

7. Singleton R, Lescher R, Gessner BD, Benson M, Bulkow $\mathrm{L}$, et al. (2015) Rickets and vitamin D deficiency in Alaska native children. J Pediatr Endocrinol Metab 28: 815-823.

8. Wheeler BJ, Dickson NP, Houghton LA, Ward LM, Taylor BJ (2015) Incidence and characteristics of vitamin $D$ deficiency rickets in New Zealand children: a New Zealand Paediatric Surveillance Unit study. Aust N Z J Public Health 39: 380-383.

9. Thacher TD, Fischer PR, Tebben PJ, Singh RJ, Cha SS, et al. (2013) Increasing incidence of nutritional rickets: a population-based study in Olmsted County, Minnesota. Mayo Clin Proc 88: 176-183.

10. Akazawa Y, Shiohara M, Amano Y, Uchida N, Nakamura $\mathrm{S}$, et al. (2010) The clinical characteristics of vitamin D deficiency in childhood: a systematic literature review of Japanese patients. J Pediatr Endocrinol Metab 23: 675684.

11. Matsuo K, Mukai T, Suzuki S, Fujieda K (2009) Prevalence and risk factors of vitamin D deficiency rickets in Hokkaido, Japan. Pediatr Int 51: 559-562.

12. Yorifuji J, Yorifuji T, Tachibana K, Nagai S, Kawai M, et al. (2008) Craniotabes in normal newborns: the earliest sign of subclinical vitamin D deficiency. J Clin Endocrinol
Metab 93: 1784-1788.

13. Kubota T, Kotani T, Miyoshi Y, Santo Y, Hirai H, et al. (2006) A spectrum of clinical presentations in seven Japanese patients with vitamin D deciciency. Clin Pediatr Endocrinol 15: 23-28.

14. Endo I, Fukumoto S, Ozono K, Namba N, Inoue D, et al. (2015) Nationwide survey of fibroblast growth factor 23 (FGF23)-related hypophosphatemic diseases in Japan: prevalence, biochemical data and treatment. Endocr $J 62$ : 811-816.

15. Hashimoto S, Fukutomi $\mathrm{K}$, Nagai $\mathrm{M}$, Nakamura $\mathrm{Y}$, Yanagawa H, et al. (1991) A method of interval estimation for number of patients in the nationwide epidemiological survey on intractable diseases. Nihon Koshu Eisei Zasshi 38: 880-883 (In Japanese).

16. Wagner CL, Greer FR (2008) Prevention of rickets and vitamin D deficiency in infants, children, and adolescents. Pediatrics 122: 1142-1152.

17. Callaghan AL, Moy RJD, Booth IW, Debelle G, Shaw NJ (2005) Incidence of symptomatic vitamin D deficiency. Arch Dis Child 91: 606-607.

18. Ward LM, Gaboury I, Ladhani M, Zlotkin S (2007) Vitamin D-deficiency rickets among children in Canada. CMAJ 177: 161-166.

19. Beck-Nielsen SS, Brock-Jacobsen B, Gram J, Brixen K, Jensen TK (2009) Incidence and prevalence of nutritional and hereditary rickets in southern Denmark. Eur J Endocrinol 160: 491-497.

20. Munns CF, Simm PJ, Rodda CP, Garnett SP, Zacharin $\mathrm{MR}$, et al. (2012) Incidence of vitamin D deficiency rickets among Australian children: an Australian Paediatric Surveillance Unit study. Med J Aust 196: 466-468.

21. Misra M, Pacaud D, Petryk A, Collett-Solberg PF, Kappy M (2008) Vitamin D deficiency in children and its management: review of current knowledge and recommendations. Pediatrics 122: 398-417.

22. Doulgeraki AE, Manousakis EM, Papadopoulos NG (2017) Bone health assessment of food allergic children on restrictive diets: a practical guide. J Pediatr Endocrinol Metab 30: 133-139.

23. Holick MF, Binkley NC, Bischoff-Ferrari HA, Gordon CM, Hanley DA, et al. (2011) Evaluation, treatment, and prevention of vitamin D deficiency: an Endocrine Society clinical practice guideline. J Clin Endocrinol Metab 96: 1911-1930. 